\title{
A New Auto-Guiding System for CQUEAN
}

\author{
Nahyun Chol ${ }^{1}$, Won-Kee Park ${ }^{2}$, Hye-In LeE ${ }^{1}$, Tae-Geun Ji ${ }^{3}$, Yiseul Jeon ${ }^{4}$, Myungshin Im ${ }^{4}$, And Soojong Pak ${ }^{1}$ \\ ${ }^{1}$ School of Space Research and Institute of Natural Sciences, Kyung Hee University, 1732 Deogyeong-daero, Giheung-gu, \\ Yongin, Gyeonggi 446-701, Korea; nahyun@khu.ac.kr, hyeinlee@khu.ac.kr, soojong@khu.ac.kr \\ ${ }^{2}$ Korea Astronomy and Space Science Institute, 776 Daedukdae-ro, Yuseong-gu, Daejeon 305-348, Korea \\ wkpark@kasi.re.kr \\ ${ }^{3}$ Department of Astronomy and Space Science, Kyung Hee University, 1732 Deogyeong-daero, Giheung-gu, Yongin, \\ Gyeonggi 446-701, Korea; jtg777@khu.ac.kr \\ ${ }^{4}$ CEOU-Astronomy Program, Department of Physics and Astronomy, Seoul National University, 1 Gwanak-ro, \\ Gwanak-gu, Seoul 151-742, Korea; ysjeon@astro.snu.ac.kr, mim@astro.snu.ac.kr
}

Received November 19, 2014; accepted April 21, 2015

\begin{abstract}
We develop a new auto-guiding system for the Camera for QUasars in the EArly uNiverse (CQUEAN). CQUEAN is an optical CCD camera system attached to the 2.1-m Otto-Struve Telescope (OST) at McDonald Observatory, USA. The new auto-guiding system differs from the original one in the following: instead of the cassegrain focus of the OST, it is attached to the finder scope; it has its own filter system for observation of bright targets; and it is controlled with the CQUEAN Auto-guiding Package, a newly developed auto-guiding program. Finder scope commands a very wide field of view at the expense of poorer light gathering power than that of the OST. Based on the star count data and the limiting magnitude of the system, we estimate there are more than 5.9 observable stars with a single FOV using the new auto-guiding CCD camera. An adapter is made to attach the system to the finder scope. The new auto-guiding system successfully guided the OST to obtain science data with CQUEAN during the test run in 2014 February. The FWHM and ellipticity distributions of stellar profiles on CQUEAN, images guided with the new auto-guiding system, indicate similar guiding capabilities with the original auto-guiding system but with slightly poorer guiding performance at longer exposures, as indicated by the position angle distribution. We conclude that the new auto-guiding system has overall similar guiding performance to the original system. The new auto-guiding system will be used for the second generation CQUEAN, but it can be used for other cassegrain instruments of the OST.
\end{abstract}

Key words: instrumentation: CCD camera — techniques: auto-guiding — telescopes

\section{INTRODUCTION}

When a telescope tracks a star during an astronomical observation, the star drifts out of the field of view unless the telescope tracking system is accurately calibrated. The auto-guiding system continually calculates the offset of the guide star and feeds back the amount of offset to the Telescope Control System (TCS), which results in reducing the error of tracking (Chen et al. 2012). As a result, a better image can be obtained than without an additional auto-guiding system or with a manual guiding method (Birney et al. 2006).

The auto-guiding system thus has been adopted by many professional telescopes, as well as many amateur astrophotograhers. For example, Mun et al. (2006) developed a telescope control software that includes an auto-guiding system for the telescope in the Kyung Hee University (KHU) observatory. Jeong et al. (1999) and Yoon et al. (2006) developed auto-guiding systems for improving the tracking accuracy of the telescope in the Seoul National University and Chungbuk National University observatory, respectively. Sung et al. (2012) suggested a new TCS to improve the tracking accuracy of the 1.8-m telescope at the Bohyunsan Optical Astron-

Corresponding Author: W.-K. Park omy Observatory. Kanzawa et al. (2006) and Iseki et al. (2008) made an effort to improve the accuracy of pointing and guiding of Subaru Telescope.

The Camera for QUasars in EArly uNiverse (CQUEAN) is an optical charge-coupled device (CCD) camera developed by the Center for the Exploration of the Origin of the Universe (CEOU). CQUEAN is more sensitive at the longer optical wavelengths and thus it is quite efficient to search for high redshift quasars in the early universe (Park et al. 2012). It has been attached to the 2.1-m Otto Struve Telescope (hereafter, OST) at McDonald observatory, Texas, USA and has performed several observational campaigns since 2010. Because CQUEAN is installed in the cassegrain focus (f/13.65), which has a characteristic of high magnification and small field of view (FOV), a focal reducer was developed in order to reduce the focal length and result in a larger FOV (Lim et al. 2013). In addition, Kim et al. (2011) made an auto-guiding system specifically for CQUEAN, because other existing auto-guiding systems for the OST could not be adapted for CQUEAN. The auto-guiding system made by Abbott (1990) was not suitable for long time exposures, and the one for the Sandiford Echelle Spectrograph cannot be used for other instruments. 
The CQUEAN system has used the PL1001E CCD camera from Finger Lakes Instrumentation (FLI) as the guide CCD camera. The pixel size of the guide CCD camera is $24 \mu \mathrm{m} \times 24 \mu \mathrm{m}$, and its pixel scale is $0.17^{\prime \prime} /$ pixel, with a FOV of $2.97^{\prime} \times 2.97^{\prime}$. The guide camera is held by an arm that can rotate around the optical axis of the telescope to find a guide star in the off-axis fields (Kim et al. 2011). The auto-guiding software, $a g d r$, originally developed for the $2.7-\mathrm{m}$ telescope in the McDonald Observatory, was modified to be used for CQUEAN and the OST.

After about three years of operation, however, the mechanical shutter of the guide CCD camera is worn out and needs to be replaced. Meanwhile, the CQUEAN system is planned to be equipped with a new filter system. Because the filter wheel for the new filter system will interfere with the existing guide CCD camera, we decided to make a new auto-guiding system. The new system is attached to the back of the finder scope. The finder scope reduces the number of observable star significantly due to much smaller diameter, but it offers very wide FOV which should compensate for the small diameter of the telescope. A new autoguiding software, the CQUEAN Auto-guiding Package (CAP), was also developed. Based on the same autoguiding method used in the Slit Camera Package (Kwon et al. 2012; Lee et al. in preparation) for the Immersion GRating INfrared Spectrograph (IGRINS; Park et al. 2014), it detects the position of a selected source on the reference image and tracks continually the positional shifts in subsequent images. The software is written in python, which will make it easy to customize, if needed.

In this paper, we describe the new auto-guiding system in detail. We examined the system requirements, such as the required exposure time of the guide CCD camera to reduce the tracking errors, and the number of observable stars under usual observation conditions. The development of the system, installation, and test results are described, and the performance of the new auto-guiding system is compared with the original autoguiding system.

\section{Auto-Guiding SyStem ReQUiREMENTS}

\subsection{Exposure Time Requirement}

The auto-guiding system continually calculates the system positional offset during an observation. The more frequently, the better the auto-guiding. The frequency of the auto-guiding depends on many factors, such as the exposure time of the scientific observation, and the performance of the telescope tracking. To calculate the auto-guiding feedback frequency, we first examined the performance of the OST tracking system. Using the CQUEAN science camera, we took 0.5 second exposures with a 1.81 second cycle time for 5 minutes without auto-guiding. The cycle time here is defined as total time that includes exposure time, data readout time and image display time.

Under perfect guiding conditions, the position of a star center would be fixed to a single pixel across a series

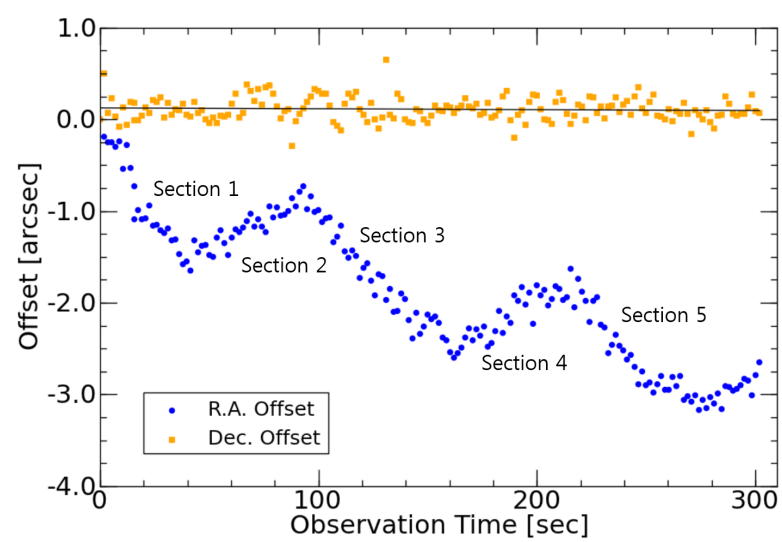

Figure 1. The drifts of the star center along RA and Dec., on the image obtained with the OST without auto-guiding. Yellow squares denote Dec. drift and blue circles drift along RA. The least square fitting result of the drifts along Dec. is shown as a line on the Dec. data. While the drift along Dec. is very small over 300 second duration, the offset along RA gets as large as $3^{\prime \prime}$, i.e., about 10 CCD pixels. Note RA offset shows a repeated reverse pattern with about 100 seconds period, which is the backlash caused by the worm gear misalignment in the OST.

of consecutive exposure images. This is usually not the case due to imperfect guiding of the telescope. Figure 1 shows the drift of the target center on the CQUEAN images along the RA, aligned with the $\mathrm{X}$ axis of the CCD chip, and Dec., Y axis of the chip, without the auto-guiding system. Kim et al. (2011) showed that there is a misalignment of the worm gear in the OST mount that causes a periodic error of about 2 minutes. The different direction of tracking offset labeled as Sections 2 and 4 are caused by this misalignment of the gear. The tracking error along Dec. turned out to be very small: $-0.0001^{\prime \prime} / \mathrm{sec}$. This value is consistent with the estimation of Kim et al. (2011). Meanwhile, the values along RA are about $0.03^{\prime \prime}$ /sec for Sections 1,3 , and 5 , and $0.02^{\prime \prime} / \mathrm{sec}$ for the other sections. Thus we estimate the average tracking error along RA to be about $0.03^{\prime \prime} / \mathrm{sec}$.

The science camera of CQUEAN has a pixel scale of about $0.3^{\prime \prime} /$ pixel with the OST. Therefore, the tracking error would not be detected if the offset amount is less than $0.3^{\prime \prime}$, i.e., one pixel. From this condition, we can estimate the cycle time of the guide camera that will produce a tracking error less than $0.3^{\prime \prime}$. Figure 1 in Kim et al. (2011) shows that telescope drift along the Dec. direction is more or less constant up to 2000 seconds. We estimated from Figure 1 that the drift along declination direction will be about $0.3^{\prime \prime}$ for a 3000 second cycle time and along RA direction about $0.3^{\prime \prime}$ for a 10 second cycle time. Therefore, we deduced that the cycle time of the guide camera should be less than 10 seconds, which is significantly longer than the shortest exposure time of the guide CCD camera. 


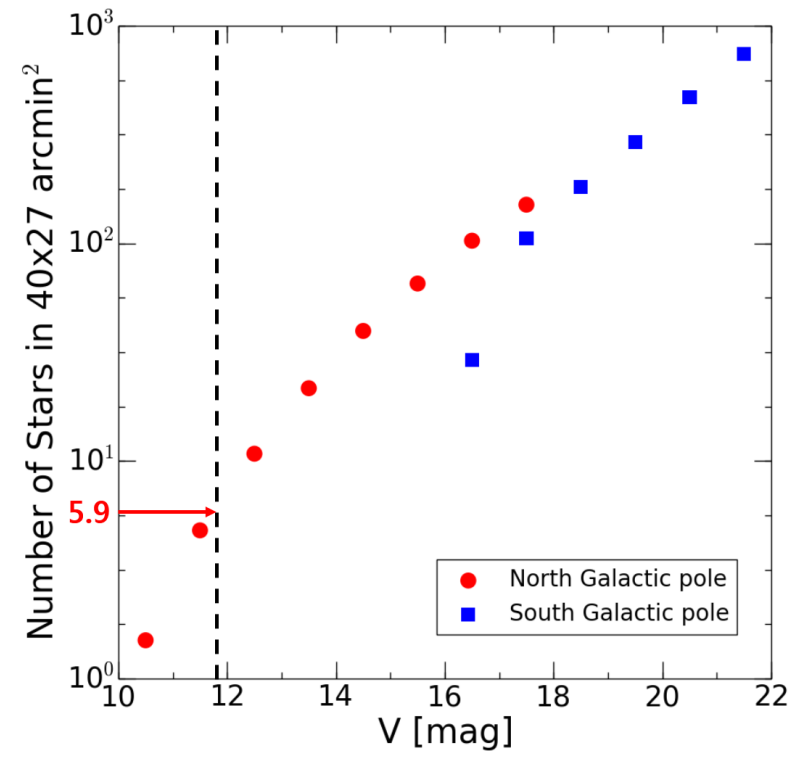

Figure 2. The number of observable guide stars in $40^{\prime} \times 27^{\prime}$ field with $\mathrm{S} / \mathrm{N}$ ratio of 10 with 1 second exposure. The red circles represent the number of stars in the north galactic pole and the blue squares the stars in the south galactic pole. Black dashed line indicates the observed limiting magnitude for the new auto-guide system.

\subsection{Expected Limiting Magnitude and Number of Guide Stars in Guide FOV}

The feasibility of the auto-guiding system also depends on the number of observable stars. We calculated the number of observable stars, i.e., brighter than the limiting magnitude of the new auto-guiding system.

The expected limiting magnitude is estimated with the formula below (McLean 2008):

$$
m=m_{z p}-2.5 \log \left(\frac{1}{g} \cdot \frac{S}{N} \cdot \frac{R}{T} \cdot \sqrt{\frac{N_{p i x}}{n_{0}}}\right)
$$

where $m_{z p}$ is the zero point, $\mathrm{g}$ is the gain in units of electron/ADU, $\mathrm{S} / \mathrm{N}$ is the signal to noise ratio, $N_{p i x}$ is the number of pixels that cover a stellar profile, $n_{0}$ is the number of exposures, $\mathrm{T}$ is the exposure time in units of second, and $\mathrm{R}$ is the readout noise in units of electrons. The zeropoint of the system is estimated from the images obtained during the test runs. It turned out to be 18.7 , with a CCD gain of 0.7 from the camera manufacturer's report. With the zeropoint estimated, the limiting magnitude of the system is calculated to be 11.7 in $V$, for the case of $\mathrm{S} / \mathrm{N}$ ratio of 10 with 1 second exposure, readout noise of 12 electrons, $N_{\text {pix }}$ of 12.2 pixels based on the pixel scale of the guide camera, and average seeing of $1.2^{\prime \prime}$ at the McDonald observatory (Kim et al. 2011).

Figure 2 shows the expected number of stars in the galactic pole regions that would fall in the FOV of our guide CCD camera, using the star count data (Allen 2000). It can be seen from the plot that there are

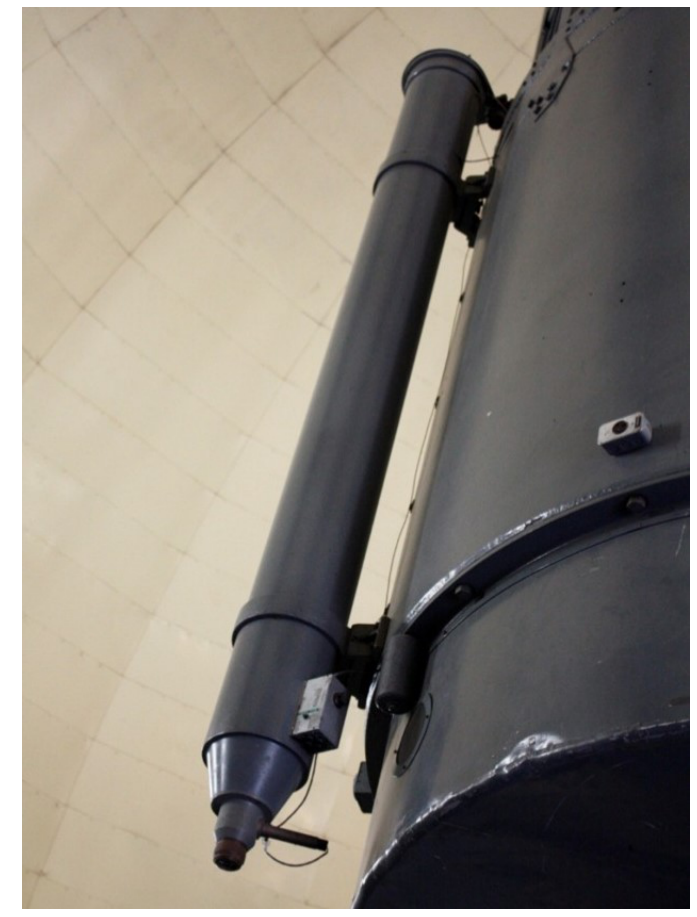

Figure 3. The finder scope attached to the OST. The picture shows the finder scope with an eyepiece. We removed the eyepiece and used an adapter to attach the auto-guiding camera and filter wheel.

about 5.9 stars that are brighter than the limiting magnitude of the system. Note that the number of observable stars will get larger in the lower galactic latitude region. Therefore, we conclude that our auto-guiding system is feasible because it can have enough number of stars for all sky regions.

\section{System Configuration}

The new auto-guiding system is attached to the finder scope of the OST. The finder scope is a refractor with a diameter of $255 \mathrm{~mm}$. Although an eyepiece was installed for visual viewing as seen in Figure 3, it has been left unused for many years since a computer controlled TCS was introduced for the OST. The complete optical configuration of the finder scope is not known except for its focal length of $3037 \mathrm{~mm}$. Figure 4 shows the auto-guiding system on the finder scope. The new auto-guiding system consists of an optical CCD camera and a filter wheel. An adapter to attach the camera and the filter wheel to the telescope was constructed. By adjusting the adapter, the camera position along the light path can be adjusted for correct focus. In addition to hardware, we also built a new software to control the new auto-guiding system. The whole configuration of the second generation CQUEAN, including the new auto-guiding system, is described as a block diagram in Figure 5. Since the guide CCD camera is now attached to the finder scope, the new auto-guiding system is physically separated from the CQUEAN system. Therefore, the new auto-guiding system can be used not 


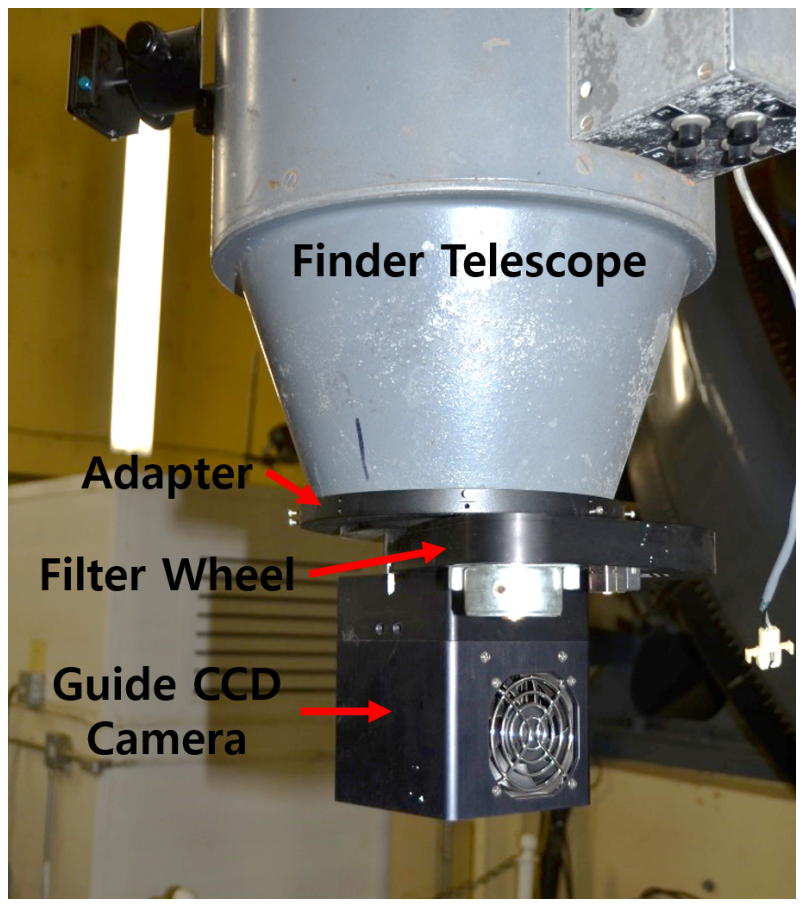

Figure 4. Picture of the auto-guide system attached to the finder scope.

Table 1

Specifications of the ML11002 CCD camera on the new guide system

\begin{tabular}{cc}
\hline Pixel Size & $9 \mu \mathrm{m} \times 9 \mu \mathrm{m}$ \\
Pixel Scale & $0.61^{\prime \prime} /$ pixel \\
Field of View & $40^{\prime} \times 27^{\prime}$ \\
CCD Type & Interline Transfer \\
Cooling method & Air Cooling \\
Linear Full Well & 40,000 e- \\
Gain & 0.7 electrons $/$ ADU \\
Readout noise & 12 e-RMS @ 1 MHz \\
Readout Speed & $1 \mathrm{MHz} @ 16$-bit data \\
Peak Quantum Efficiency & $50 \%$ @ 500 nm \\
\hline
\end{tabular}

*Measured by the authors.

only with CQUEAN, but also with other instruments attached to the OST. With a little further modification in the TCS interface part of the software, which is quite easy since the software is written in python, we expect that the auto-guiding system can be used for other telescope systems as well. In this section, each component of the new auto-guiding system is described in detail.

\subsection{Guide Camera and Filter Wheel}

One of the requirements for the guide CCD camera is that it should be small and light enough to be attached to the back of the finder scope. The previous autoguiding system consists of a guide CCD camera, an arm to hold the camera, and a motor drive which rotates the arm around the on-axis field of the OST. The OST itself is very large and heavy enough to hold all these auto-guiding hardware in stable condition. But the finder scope is much smaller and lighter so that its

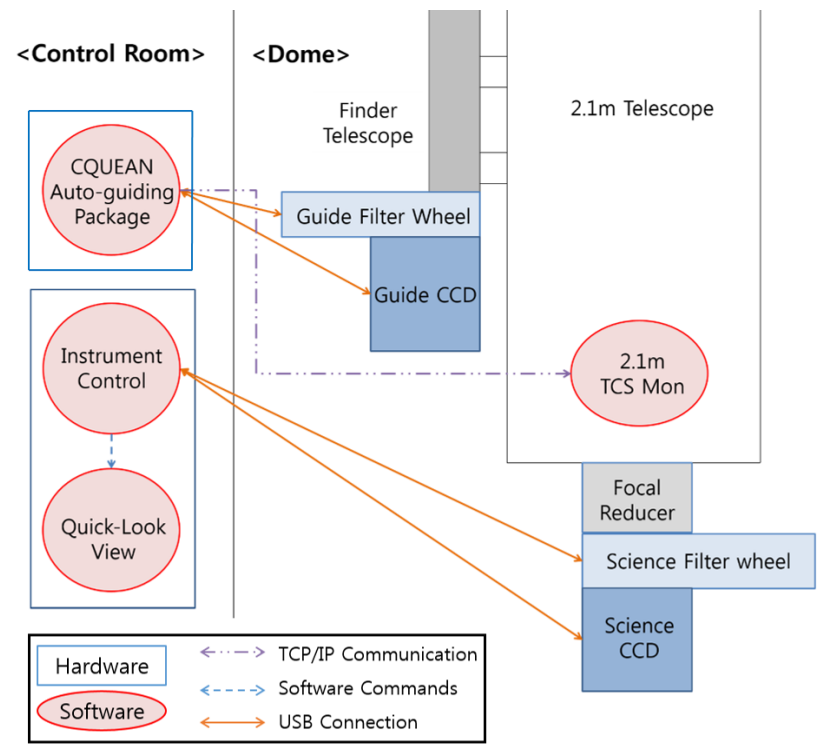

Figure 5. Block diagram of new CQUEAN system.

weight balance can be easily affected even though a motor and arm are no longer needed in the new system. Another requirement for the guide CCD camera is that it does not have a mechanical shutter. Auto-guiding systems usually take enormous amounts of short exposures in a night to track the guide star. According to the camera manufacturer, the Mean Time Between Failure (MTBF) of the shutter for PL1000E CCD camera in the previous auto-guiding system is 1000000 . While this number looks very large, the shutter was worn out after about three years of operation. Therefore, we want the guide CCD camera to be free of a mechanical shutter to increase its operation lifetime. Based on these requirements, we chose the ML11002 model among MicroLine series from FLI as the new guide CCD camera. The MicroLine series comes with a smaller and lighter housing than the ProLine series. The model has an interline type Kodak KAI-11002 chip. Interline type CCDs have separate readout channels between the light-sensitive pixels, and they can read the data out during exposures. Therefore, unlike a frame transfer type CCD, they do not require a shutter during readout. The low fill factor of the interline type CCD results in poorer sensitivity than the frame transfer type, but auto-guiding usually requires the observation of bright targets. The CCD chip in the ML11002 camera has $4008 \times 2672$ pixels of $9 \mu \mathrm{m} \times 9 \mu \mathrm{m}$ size. The pixel scale is $0.61^{\prime \prime} /$ pixel, and the FOV is $40^{\prime} \times 27^{\prime}$ at the finder scope. Table 1 shows the relevant characteristics of our guide CCD camera. The $\mathrm{CCD}$ is cooled down to $-10^{\circ} \mathrm{C}$ during the operation by built-in fan cooling. It is connected to the computer via Universal Serial Bus (USB). An USB repeater is used to overcome the short distance limitation of the USB connection. Figure 6 shows an image obtained with guide the CCD camera, along with a box indicating the FOV of CQUEAN science camera.

A filter wheel is installed in front of the guide CCD camera for possible science observations. A CFW-1-5 


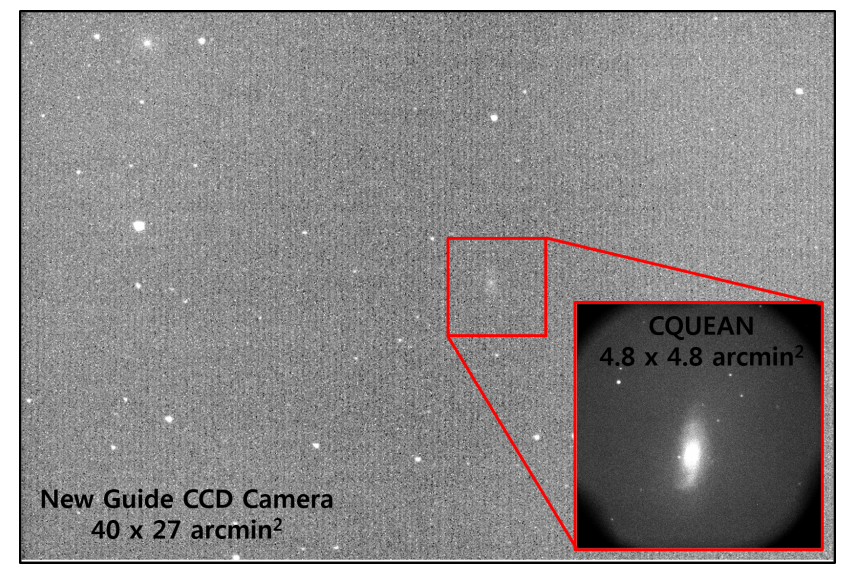

Figure 6. An example image obtained with the guide CCD camera. The FOV of the guide CCD camera is $40^{\prime} \times 27^{\prime}$. The $4.8^{\prime \prime} \times 4.8^{\prime \prime}$ FOV of the science CCD camera is shown as red box in the image.

model, also made by the same company, is used in our system. The filter wheel is also connected to the control computer via USB. This filter wheel can house up to five circular filters with a diameter of $50 \mathrm{~mm}$ and thickness less than $5 \mathrm{~mm}$. We installed only four filters: Johnson $B, V, R$ and $I$, and left the remaining hole empty. During the normal auto-guiding operation mode, we do not use any filter for maximum transmission efficiency. But for bright targets such as supernova or variable stars in the Galaxy, the guide CCD camera can carry out a science observation on its own, or along with CQUEAN science camera which has different filters. Although we will not present any additional details about the scientific aspect of the guide CCD camera system in this study, further tests are planned to calibrate the system for scientific observations.

To attach the camera and the filter wheel to the finder scope, we designed and fabricated a custom adapter. Figure 7 shows the picture and the front view drawing of the adapter. The adapter is made of aluminum, and all parts of the adapter were anodized to prevent the reflection of light. The adapter basically consists of two plates and a ring. One plate holds the guide CCD camera system, and the other one is attached to the telescope. A large ring holds the two plates securely to each other. By rotating the ring, the distance between the two rings can be adjusted for fine focus adjustment of the guide CCD camera. Once the camera is in focus, the position is fixed by 8 bolts around the ring.

\subsection{Guiding Software}

CAP controls the guide CCD camera and the filter wheel to obtain the imaging data from the guide CCD camera system, and interacts with the TCS of the OST via TCP/IP protocol to control the telescope movement. It is designed and built based on the auto-guiding software for the IGRINS slit-viewing camera. However, the user interface was designed to be similar to that of $a g d r$, the original auto-guiding software, so that an experienced CQUEAN user has little difficulty to use the

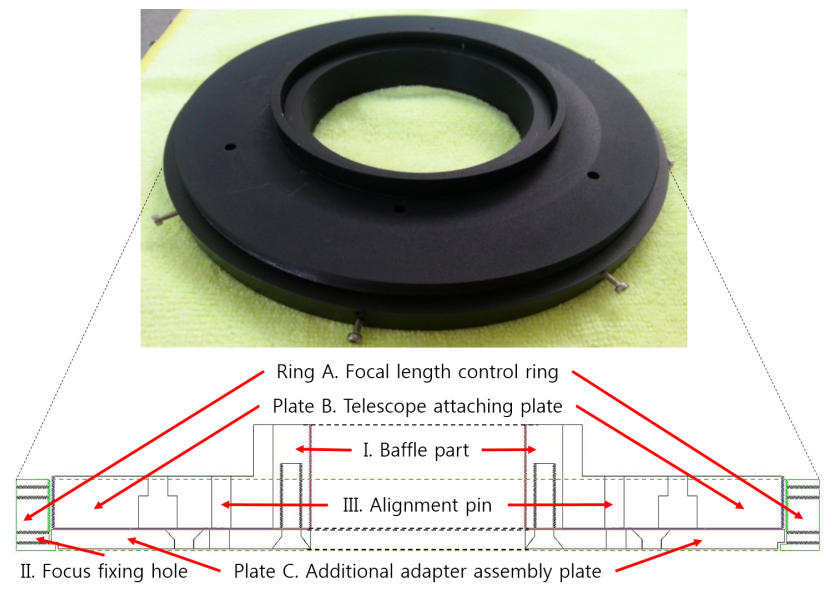

Figure 7. Picture and front view drawing of the adapter. The adapter consists of three parts: two plates indicated by $\mathrm{B}$ and $\mathrm{C}$, and a ring (marked as $\mathrm{A}$ ) that holds the two plates. The baffle part, marked as $\mathrm{I}$, in the plate $\mathrm{B}$ is inserted into the baffle of the finder scope. The plate $\mathrm{C}$ is holding the filter wheel. The alignment pin III aligns the two plates B and $\mathrm{C}$. By rotating the ring $\mathrm{A}$, the distance between the two rings can be adjusted for better focus of the camera system. 8 bolts are used in part II to fix the distance after the system is in focus.

new software. CAP is written in python. The Tkinter library is used for its graphical user interface (GUI) design, and the mathematical library numpy for the data analysis code. It is running on a linux platform.

Figure 8 shows the GUI of the CAP. The main panel of the CAP is the Image Window, which shows a full image of the guide CCD camera. When a user selects a guide star by clicking on a star in the Image Window, a yellow box appears to indicate that a guide star has been selected, and a zoomed image of the star appears in the Zoom-in Box panel. Full Width at Half Maximum (FWHM) of the guide star is obtained with Gaussian profile fitting on the selected guide star. In addition, the observation field of the CQUEAN science camera is indicated with a purple box in the Image Window and CQUEAN panel. The camera panel has the following camera control functions: Initialization, setting CCD temperature, showing current temperature, exposure time, $\mathrm{X}-\mathrm{Y}$ binning, filter, and observation start/stop buttons. $\mathrm{X}$ and $\mathrm{Y}$ in the guide panel represent the current position of the guide star and $\mathrm{dx}$ and dy the position difference between the current image and the reference image. The reference image is the one that the user has selected as the current guide star. The TCS panel displays the current pointing of the OST and its focus value. It also has four arrow buttons to move the OST by 10 arcseconds on the sky. Finally, the CAP status window shows low level messages so that a user can check the cause of a problem in the case of an emergency.

The guide camera takes an image of the field and displays it in the Image window. After the user chooses a guide star with a click on the image, CAP automatically puts a yellow box around it. CAP finds the $\mathrm{X}$ and $\mathrm{Y}$ 


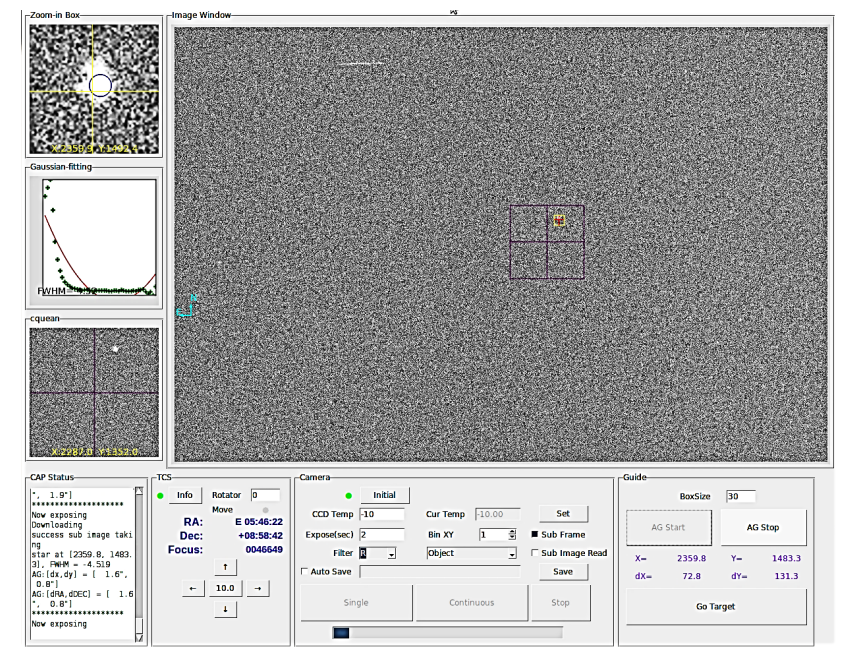

Figure 8. The graphical user interface of CAP, the autoguiding software.

coordinates of the guide star center using 1-D Gaussian fitting. The auto-guiding starts when the user clicks the AG Start button, by continually making exposures with exposure time set within the program. The autoguiding system calculates the guide star center coordinates in each frame and the offset with respect to the reference image. The CAP converts the offset into celestial coordinates based on the current OST position obtained from the TCS. Then the offset information is sent to the TCS and the TCS moves the telescope to correct for the tracking error. This procedure is repeated until the user stops it.

\section{INSTALLATION AND TESTING}

The first hardware test of the new auto-guiding system occurred in 2013 June, when the guide CCD camera was installed at the finder scope for the first time. This test was to verify that all hardware components were assembled as expected and check the focus adjustment with the new adapter. The optimum focus positions depend on the filters, because of the chromatic aberration of the telescope.

Additional test observations were carried out to find the limiting magnitude of the system at the finder scope. We acquired 50 second exposure images of V1515 Cyg with $B, V, R$ and $I$ band filters. The limiting magnitude was estimated to be 11.7 in $V$. Note that the actual value will be higher (i.e., fainter) than this because the test observations were conducted during a non-photometric night.

The movement of the OST is controlled in celestial coordinate units while the CAP obtains the tracking offset in pixel units. Therefore the pixel scale of the guide CCD camera is very important for accurate autoguiding. The pixel scale of the guide CCD camera with the finder scope was accurately calibrated with the imaging data obtained in the next test run in October 2013. The CAP code was revised with the measured pixel scale.

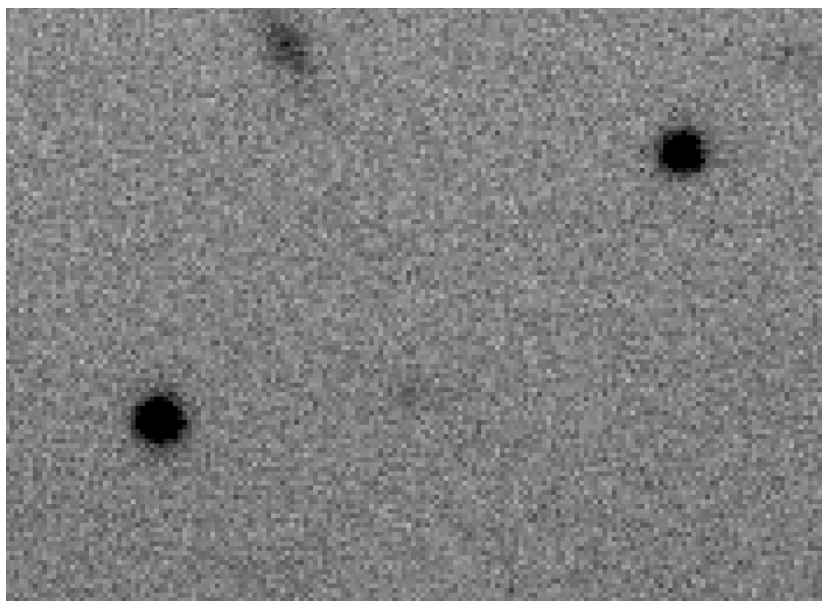

Figure 9. Part of a 300 second CQUEAN science image obtained with CAP guiding. The seeing was $1.4^{\prime \prime}$.

The instrument control and TCS communication part of the CAP were tested on subsequent nights in the same run. CAP successfully controlled the filter wheel and the camera to take the image data. The user can set the camera exposure time, $\mathrm{X}$ and/or $\mathrm{Y}$ binning, CCD cooling, and so on within the CAP software. Communication between CAP and the TCS, sending and receiving the information of $\mathrm{RA}$ and Dec. was also confirmed during this test run.

The real auto-guiding test with CAP was carried out on three test runs in 2013 November, 2014 February, and 2014 April. The full sequence of auto-guiding procedures was tested, and new the auto-guiding system was confirmed to work without any problem. Autoguiding tests were performed using various configurations such as with different guide CCD exposure times, different regions in sky, and for different exposure times of the science CCD camera. For comparison, autoguiding tests with both the new and the original systems were also carried out.

\section{Discussion}

\subsection{Guiding Performance of CAP}

The performance of the auto-guiding system can be estimated by examining the stellar profiles on science camera images that are obtained using the auto-guiding system. Figure 9 shows a section of a 300 second CQUEAN image, where two stellar images are seen. Both stellar images are of circular shape, indicating the guiding during the exposure was quite good. It would be better to compare with the same exposure image obtained without auto-guiding, but it could not be obtained during the test. Instead, we refer to Figure 10 in Kim et al. (2011) for the performance of the OST tracking without auto-guiding.

To estimate the guiding performance of the new autoguiding system in a more quantitative way, we measured the stellar profile parameters such as FWHM, ellipticity, and position angle (PA) with Source Extractor (Bertin \& Arnouts 1996) on several science CCD im- 


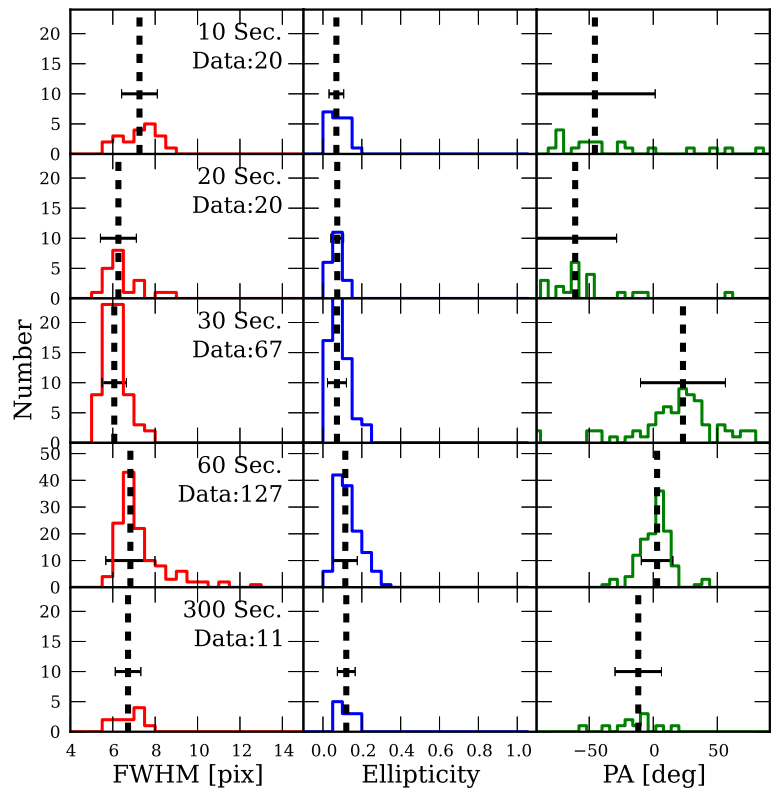

Figure 10. Distributions of the FWHM (left column), ellipticity (middle column) and PA (right column) of stellar image profiles on CQUEAN science image auto-guided with CAP. The results for 10, 20, 30, 60 and 300 second exposures are shown in each row of the panels. The vertical dashed line in each panel indicates the median value of the parameters shown, and the horizontal bar indicates the standard deviation $(\sigma)$ of each parameter distribution. See the text for details.

ages that were obtained with the auto-guiding system, CAP. We also obtained the same exposure images autoguided by agdr, the previous auto-guiding system, and measured the same stellar profile parameters. To ensure similar test conditions, we first obtained a series of science images auto-guided with agdr, then we repeated the same target observations auto-guided with CAP. The images of CQUEAN science camera were obtained with 10, 20,30, 60, and 300 seconds, and the exposure times of both guide CCD cameras were set to 1 second during all test observations. Table 2 lists the number of CQUEAN images used in this measurements along with their filters. Note that some images were obtained with different filters from $Y$-band because all data were obtained during the course of science observations. Filters had to be changed according to the science targets so it was not possible to obtain all test data sets with the same filter. To avoid the coma aberration effect, only the point sources of stellarity $>0.8$ and located within a radius of 350 pixels from the CQUEAN image center were examined. The FWHM of the profile will be in general larger in a more poorly guided image than that on a better guided image. The ellipticity is zero for a perfect round profile, and becomes 1 a line. The PA is the angle between the major axis of the stellar profile and the $\mathrm{X}$ axis of the science camera image, and it ranges between $-90^{\circ} \sim 90^{\circ}$. The stellar profile would be an ellipse rather than a circle on a poorly guided image,
Table 2

Description of the image sets used for profile examination.

\begin{tabular}{cccccc}
\hline & \multicolumn{2}{c}{ CAP } & & \multicolumn{2}{c}{$a g d r$} \\
\cline { 2 - 3 } \cline { 5 - 6 } Exp. time & Image \# & Filter & & Image \# & Filter \\
\hline 10 & 10 & $Y$ & & 10 & $Y$ \\
20 & 10 & $Y$ & & 10 & $Y$ \\
30 & 15 & $I z$ & & 15 & $I z$ \\
60 & 30 & $I s$ & & 30 & $I s$ \\
300 & 2 & $Y$ & & 2 & $Y$ \\
\hline
\end{tabular}

thus ellipticity of all stellar profiles would be close to 1 . The PA distribution would have a strong peak toward the star drifting direction which corresponds to the $\mathrm{X}$ axis of the chip in our case. On the other hand, the ellipticities would be all zero and the PA distribution would be uniform on a perfectly guided image.

The analysis results are plotted in Figures 10 and 11. As seen in Figure 10, the median value of FWHM remains more or less constant with respect to the exposure time for CAP case. As for the agdr case (Figure 11), a similar trend was observed. On the $60 \mathrm{sec}-$ ond images, the distribution of FWHMs of the images guided with CAP is more concentrated on a smaller value than those on the image obtained with agdr. This, however, it does not mean that the CAP guiding performance is better than agdr since FWHM alone cannot represent the guiding performance. The longest exposure might show the difference of guiding performance more clearly, but it was not evident as only a few 300 second images were acquired.

As for the ellipticity, the median values remain more or less constant with respect to the exposure times in both cases. The median values of the ellipticity turn out to be around $0.1 \sim 0.2$, indicating the stellar profiles to be almost circular in both cases. We note that the medians of ellipticity produced by agdr are slightly lower than those produced by CAP hinting that agdr guiding produced more circular stellar profiles on the CQUEAN images, but the difference is smaller than the uncertainty. The PA distribution on the 60 second images guided with CAP shows the stronger peak at around $0^{\circ}$ than with $a g d r$. This indicates that higher fraction of stars on CAP guided images have elongated profiles along the $\mathrm{X}$ axis, the tracking direction. Note that the 10 second and 20 second image cases seem to show the opposite trend such that CAP guiding is better than agdr guiding. But these features seem to be fictitious as the PA distributions peak at around $-40^{\circ}$, which is unrealistic. There are only eleven data points available for the 300 second exposure case, so no concrete conclusion could be drawn from this data set. However, judging from the two exposure cases of 30 seconds and 60 seconds, we guess that the PA distribution of the CAP guiding would show an even stronger concentration than agdr.

The analysis of the PA distributions indicates that CAP has poorer guiding performance for longer exposures than 60 seconds. This PA distribution result 


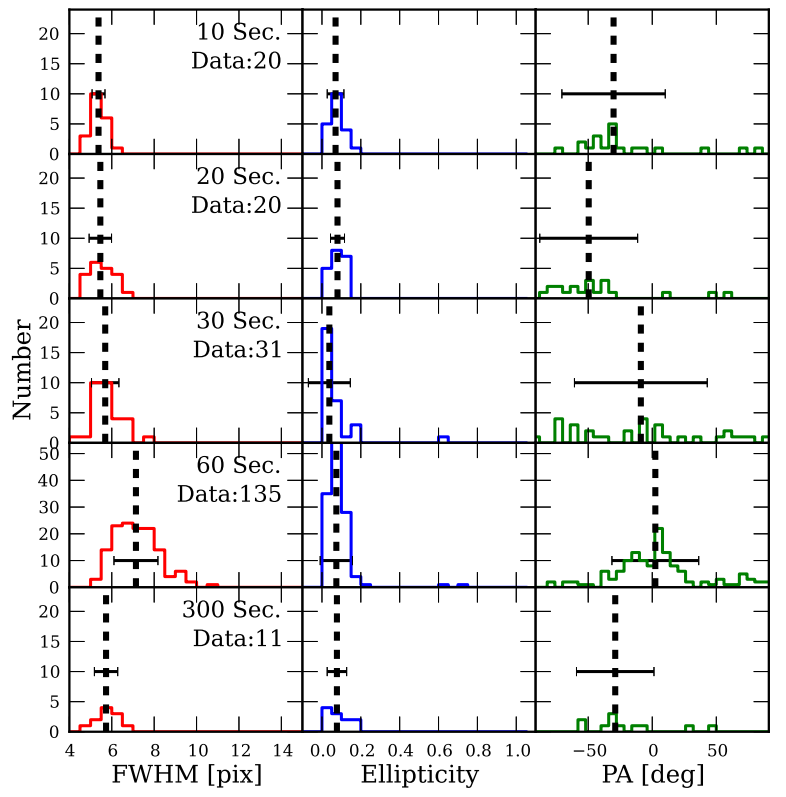

Figure 11. Same as Figure 10 but for CQUEAN images autoguided with $a g d r$.

seems to be due to the larger pixel scale of the new auto-guiding camera, $0.61^{\prime \prime} / \mathrm{pix}$, which is almost double that of the CQUEAN science camera, $0.281^{\prime \prime} /$ pix. The larger pixel scale of the guide camera means that the stellar profile center might wander within two pixels on the science image even if the CAP perfectly guided the telescope. However, the FWHM and ellipticity distribution show very similar shape to those obtained with agdr, so the effect from the guiding performance difference does not appear substantial. Also, since CQUEAN has been observing mainly point sources, the quasar candidates, the degradation of the resultant profile quality by about a pixel at most would not affect the science performed with CQUEAN significantly. Equation (1) shows that the limiting magnitude of the CQUEAN system is proportional to the logarithm of the FWHM, since $N_{p i x}$ is proportional to the square of the FWHM. If we assume that the CAP guiding may add at most one pixel to the resulting FWHM compared to $a g d r$, we can estimate the limiting magnitude change of the system with respect to the guiding performance. The change in limiting magnitude is calculated to be -0.27 magnitude (i.e., brighter) for a seeing of $1.0^{\prime \prime}$, and -0.14 magnitude for $2.0^{\prime \prime}$. The larger the seeing the smaller the degradation of CQUEAN performance. Considering all these factors, we conclude that the new auto-guiding system meets the telescope tracking requirements of the second generation CQUEAN.

\subsection{Cycle Time Effects}

The effect of cycle time, i.e. the exposure time of the guide camera, on the telescope tracking is investigated in a more quantitative way. During the 2014 February run, we observed the FU Ori field with CQUEAN
Table 3

Auto-guiding offset as function of cycle time.

\begin{tabular}{cccc}
\hline $\begin{array}{c}\text { Cycle } \\
\text { time }\end{array}$ & $\begin{array}{c}\text { Exposure } \\
\text { time }\end{array}$ & $\sigma{\text { (RA offset })^{\dagger}}$ & $\sigma{\text { (Dec. offset })^{\dagger}}^{\dagger}$ \\
\hline 3.6 & 2 & 0.88 & 0.66 \\
4.6 & 3 & 0.87 & 0.64 \\
6.4 & 5 & 0.93 & 0.36 \\
31.5 & 30 & 1.03 & 0.95 \\
\hline
\end{tabular}

${ }^{\dagger}$ In units of CQUEAN science CCD camera pixels.

auto-guided with CAP. We guided the telescope with four different cycle times. During each cycle time observation, the offsets of a target with respect to the reference frame along the RA and Dec. directions were investigated, and the result is shown in Table 3 . The measured offsets for cycle times of 3.6, 4.6, and 6.4 second cycle times have standard deviation $\sigma$ less than 1 pixel of the CQUEAN science camera. This is not the case for a 31.5 second cycle time, which has $\sigma$ larger than 1 pixel along the $\mathrm{X}$ direction. This would cause the stellar profile to spread out on more pixels on the science CCD. This result shows cycle time should be less than 31.5 seconds. This is consistent with the expected cycle time of 10 seconds obtained in Section 2.1. Therefore, it is recommended that the observer set the cycle time of the guide CCD camera to be less than 10 seconds.

\section{Summary ANd Conclusions}

We developed a new auto-guiding system for the second generation CQUEAN. Analysis of the system requirements indicated that the OST tracking can be corrected with an auto-guiding system with a cycle time shorter than 10 seconds and that the camera FOV with the finder scope is wide enough to find more than 5.9 stars that are brighter than the limiting magnitude in the case of a 1 second exposure.

The hardware of the new auto-guiding system consists of an optical CCD camera and a filter wheel. Johnson $B V R I$ filters were installed in the filter wheel for possible science observation with the guide CCD camera. This system is attached to the finder scope of the OST at the McDonald Observatory in Texas, USA. An adapter was made to attach the camera and the filter wheel to the finder scope. This adapter enables us to adjust the camera position for best focus. We also developed our own auto-guiding software, CAP, written in Python.

The new auto-guiding system was put to first full auto-guiding test on 2014 February, after several stages of partial integration and operation tests. Resultant CQUEAN images were analyzed to obtain the image profile parameters such as FWHM, ellipticity and position angles for several point sources in science images. The position angle distribution of the stellar profiles in the CQUEAN images guided with the new auto-guiding system show more concentrated distributions at around $0^{\circ}$ than those obtained with the original auto-guiding 
system, agdr, indicating slightly poorer guiding performance for the new system. However, the resultant stellar profiles are showing similar FWHM, and ellipticity distributions. The CQUEAN limiting magnitude change will be 0.27 magnitude brighter for $1.0^{\prime \prime}$ seeing condition, and 0.14 magnitude for $2.0^{\prime \prime}$. We estimated there would be no adverse effect on the science with CQUEAN images.

Based on these results, we consider the new autoguiding system to meet the tracking requirements of the CQUEAN, and thus the second generation CQUEAN as well. Moreover, since the new system is physically separated from the CQUEAN science camera system, it can work with other instruments on the OST. With a few further modifications, we expect that the new auto-guiding software package can be used for other telescopes as well.

\section{ACKNOWLedgments}

This work was supported by the National Research Foundation of Korea (NRF) grant, No. 2008-0060544, funded by the Korean government (MSIP). This study is based on data taken at The McDonald observatory of The University of Texas at Austin. The authors would like to thank Sami K. Solanki and Ho Jin for useful discussions.

\section{REFERENCES}

Abbott, T. 1990, An Off-the-Shelf Autoguider for McDonald Observatory, Proc. SPIE, 1235, 770

Allen, C. W. 2000, Allen's Astrophysical Quantities, ed. A. N. Cox (New York: Springer), 480

Bertin, E., \& Arnouts, S. 1996, SExtractor: Software for Source Extraction, A\&AS, 117, 393

Birney, D. S., Gonzalez, G., \& Oesper, D. 2006, Observational Astronomy (New York: Cambridge University Press), 230
Chen, L., Zhang, Z., \& Wang, H. 2012, The Improvement of CCD Autoguiding System for $2.5 \mathrm{~m}$ Telescope, Proc. SPIE, 8451, 84512K

Kim, E., Park, W.-K., Jeong, H., et al., 2011, Auto-Guiding System for CQUEAN (Camera for QUasars in EArly uNiverse), JKAS, 44, 115

Iseki, A., Tomono, D., Tajitsu, A., Itoh, N., \& Miki, S. 2008, Improved Guiding Accuracy through Slit Viewer of Subaru Telescope, Proc. SPIE, 7019, 70192D

Jeong, W., Lee, S.-G., \& Pak, S. 1999, Development of Control Softwares for Improvement of Tracking Efficiency of the 16 inch Telescope of Seoul National University Observatory, PKAS, 14, 47

Kanzawa, T., Tomono, D., Usua, T., Takato, N., Negishi, S., Sugahara, S., \& Itoh, N. 2006, Improvement of the Pointing Accuracy of the Subaru Telescope by Suppressing Vibrations, Proc. SPIE, 6267, 62673J

Kwon, B. Y., Kang, W., Cho, E., et al. 2012, Development of IGRINS Control Software, KNOM Review, 15, 25

Lim, J., Chang, S., Pak, S., Kim, Y., Park, W.-K., \& Im, M. 2013, Focal Reducer for CQUEAN (Camera for QUasars in EArly uNiverse), JKAS, 46, 161

McLean, I. 2008, Electronic Imaging in Astronomy (Chichester: Praxis Publishing Ltd.), 343

Mun, B.-S, Kim, S.-J., Jang, M., Min, S.-W., Seol, K.-H., \& Moon, K.-S. 2006, Development of the Software for 30 Inch Telescope Control System at KHAO, PKAS, 21, 81

Park, C., Jaffe, D. T., Yuk, I. -S., et al., 2014, Design and Early Performance of IGRINS (Immersion Grating Infrared Spectrometer), Proc. SPIE, 9147

Park, W.-K., Pak, S., Im, M., et al. 2012, Camera for QUasars in EArly uNiverse (CQUEAN), PASP, 124, 839

Romanishin, W. 2006, An Introduction to Astronomical Photometry Using CCDs, University of Oklahoma, 17

Sung, H.-I., Park, Y.-H., Lee, S.-M., Lee, B.-C., Seong, H.C., \& Oh, H.-L. 2012, The Status and Improvement Plan of $1.8 \mathrm{~m}$ Telescope Control system at BOAO, PKAS, 27, 95

Yoon, J.-N., Lee, C.-U., Cha, S.-M., \& Kim, Y. 2006, A Construction of an Automatic Observation System for Bright and Long Period Variable Stars, JA\&SS, 23, 143 